Supplement of Atmos. Chem. Phys., 16, 14545-14562, 2016

http://www.atmos-chem-phys.net/16/14545/2016/

doi:10.5194/acp-16-14545-2016-supplement

(C) Author(s) 2016. CC Attribution 3.0 License.

(c) (i)

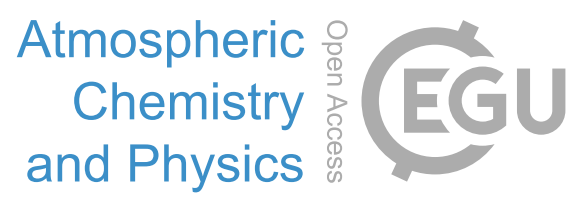

Supplement of

\title{
Inventory of anthropogenic methane emissions in mainland China from 1980 to 2010
}

Shushi Peng et al.

Correspondence to: Shushi Peng (speng@ @ pku.edu.cn)

The copyright of individual parts of the supplement might differ from the CC-BY 3.0 licence. 
Table S1. Proxy data collected for mapping spatial distributions of $\mathrm{CH}_{4}$ emissions for each source sector.

\begin{tabular}{|c|c|c|c|}
\hline & Proxy data & $\begin{array}{c}\text { Spatial } \\
\text { resolution }\end{array}$ & Reference \\
\hline Livestock & $\begin{array}{l}\text { Gridded data for numbers of animals in } \\
\qquad 2005\end{array}$ & $1 \mathrm{~km} \times 1 \mathrm{~km}$ & Robinson et al. (2011) \\
\hline Rice cultivation & Gridded harvested area of rice & $0.083^{\circ} \times 0.083^{\circ}$ & Monfreda et al. (2008) \\
\hline $\begin{array}{l}\text { Biomass and } \\
\text { biofuel burning }\end{array}$ & Gridded rural population in 2010 & $0.1^{\circ} \times 0.1^{\circ}$ & $\begin{array}{c}\text { China statistical } \\
\text { yearbook for regional } \\
\text { economy (2011) }\end{array}$ \\
\hline Coal exploitation & $\begin{array}{c}\text { Annual production from } 4264 \text { coal } \\
\text { production sites }\end{array}$ & $0.1^{\circ} \times 0.1^{\circ}$ & Liu et al. (2015) \\
\hline Oil and gas systems & EDGARv42 gridded 1B2a subcategory & $0.1^{\circ} \times 0.1^{\circ}$ & $\begin{array}{c}\text { EDGARv42; Schwietzke } \\
\text { et al. (2014) }\end{array}$ \\
\hline FF combustion & Gridded GDP in 2005 and 2010 & $1 \mathrm{~km} \times 1 \mathrm{~km}$ & Huang et al. (2014) \\
\hline Landfills & Gridded total population in 2005 and 2010 & $1 \mathrm{~km} \times 1 \mathrm{~km}$ & Huang et al. (2014) \\
\hline Wastewater & Gridded total population in 2005 and 2010 & $1 \mathrm{~km} \times 1 \mathrm{~km}$ & Huang et al. (2014) \\
\hline
\end{tabular}

\section{Reference}

China statistical yearbook for regional economy, 2010, China Statistics Press, Beijing.

Emission Database for Global Atmospheric Research (EDGAR): EDGARv4.2FT2012, European Commission, Joint Research Centre (JRC)/PBL Netherlands Environmental Assessment Agency. Emission Database for Global Atmospheric Research (EDGAR), release version 4.2. http://edgar.jrc.ec.europe.eu, 2014 (accessed Dec. 20, 2015).

Huang, Y. H., Jiang, D. and Fu, J. Y.: 1 km grid GDP data of China $(2005,2010)$, Acta Geographica Sinica, 69, 140-143, doi: 10.3974/geodb.2014.01.07.v1, 2014.

Liu, Z., Guan, D., Wei, W., Davis, S. J., Ciais, P., Bai, J., Peng, S., Zhang, Q., Hubacek, K., Marland, G., Andres, R. J., Crawford-Brown, D., Lin, J., Zhao, H., Hong, C., Boden, T. A., Feng, K., Peters, G. P., Xi, F., Liu, J., Li, Y., Zhao, Y., Zeng, N., and He, K.: Reduced carbon emission estimates from fossil fuel combustion and cement production in China, Nature, 524, 335-338, 10.1038/nature14677, 2015.

Monfreda, C., Ramankutty, N., and Foley, J. A.: Farming the planet: 2. Geographic distribution of crop areas, yields, physiological types, and net primary production in the year 2000 , Global Biogeochemical Cycles, 22, Gb1022, 10.1029/2007GB002947, 2008.

Schwietzke, S., Griffin, W. M., Matthews, H. S., and Bruhwiler, L. M. P.: Global Bottom-Up Fossil Fuel Fugitive Methane and Ethane Emissions Inventory for Atmospheric Modeling, ACS Sustainable Chemistry \& Engineering, 2, 1992-2001, 10.1021/sc500163h, 2014.

Robinson, T.P., Thornton, P.K., Franceschini, G., Kruska, R.L., Chiozza, F., Notenbaert, A., Cecchi, G., Herrero, M., Epprecht, M., Fritz, S., You, L., Conchedda, G. and See, L. (2011) Global Livestock Production Systems. FAO and ILRI, Rome. ISBN 978-92-5-107033-8. Available at: http://www.fao.org/docrep/014/i2414e/i2414e00.htm 
Table S2. IPCC default EFs.

\begin{tabular}{|c|c|c|c|c|c|}
\hline \multirow[b]{2}{*}{ Source Sector } & & \multirow[b]{2}{*}{ Unit } & \multicolumn{3}{|l|}{ EFs } \\
\hline & & & Average & Low & High \\
\hline \multirow[t]{2}{*}{ Rice cultivation } & Without organic input & $\begin{array}{l}\mathrm{g} \mathrm{CH}_{4} \mathrm{~m}^{-2} \text { day }^{-} \\
1\end{array}$ & 0.12 & 0.08 & 0.23 \\
\hline & With organic input & $\begin{array}{l}\mathrm{g} \mathrm{CH}_{4} \mathrm{~m}^{-2} \text { day }^{-} \\
1\end{array}$ & 0.25 & 0.16 & 0.57 \\
\hline \multirow{4}{*}{$\begin{array}{l}\text { Biomass and biofuel } \\
\text { burning }\end{array}$} & Crop residues burnt in household & $\mathrm{kg} \mathrm{CH}_{4} \mathrm{t}^{-1}$ & 2.7 & 2.7 & 2.7 \\
\hline & $\begin{array}{l}\text { Disposed crop residues burnt in open } \\
\text { field }\end{array}$ & $\mathrm{kg} \mathrm{CH}_{4} \mathrm{t}^{-1}$ & 2.7 & 2.7 & 2.7 \\
\hline & Firewood burnt in household & $\mathrm{kg} \mathrm{CH}_{4} \mathrm{t}^{-1}$ & 6.1 & 1.8 & 10.4 \\
\hline & Burning efficiency & - & 0.8 & 0.8 & 0.9 \\
\hline \multirow[t]{4}{*}{ Coal mining } & Underground mines: Mining & $m^{3} t^{-1}$ & 18 & 10 & 25 \\
\hline & Underground mines: Post-mining & $m^{3} t^{-1}$ & 2.5 & 0.9 & 4.0 \\
\hline & Surface mines: Mining & $m^{3} t^{-1}$ & 1.2 & 0.3 & 2.0 \\
\hline & Surface mines: Post-mining & $m^{3} t^{-1}$ & 0.1 & 0.0 & 0.2 \\
\hline \multirow[t]{2}{*}{ Oil and gas systems } & Oil & $\mathrm{kg} \mathrm{CH}_{4} \mathrm{~m}^{-3}$ oil & 4.3 & 2.1 & 18.9 \\
\hline & Gas & - & $2.0 \%$ & $0.2 \%$ & $6.2 \%$ \\
\hline \multirow{3}{*}{$\begin{array}{l}\text { Fossil fuels } \\
\text { combustion }\end{array}$} & Coal & $\mathrm{kg} \mathrm{CH}_{4} \mathrm{TJ}^{-1}$ & 1 & 0.4 & 1.6 \\
\hline & Oil & $\mathrm{kg} \mathrm{CH}_{4} \mathrm{TJ}^{-1}$ & 3 & 1.2 & 4.8 \\
\hline & Natural gas & $\mathrm{kg} \mathrm{CH}_{4} \mathrm{TJ}^{-1}$ & 1 & 0.4 & 1.6 \\
\hline \multirow[t]{6}{*}{ Landfills } & $\mathrm{k}$ in Eq. 3 & - & 0.09 & 0.08 & 0.1 \\
\hline & $T_{L}$ in Eq. 3 & years & 15.40 & 17.32 & 13.86 \\
\hline & DOC in Eq. 3 & $\%$ & 15 & 12 & 18 \\
\hline & $\mathrm{DOC}_{\mathrm{d}}$ in Eq. 3 & - & 0.5 & 0.4 & 0.6 \\
\hline & $f$ in Eq. 3 & - & 0.5 & 0.5 & 0.6 \\
\hline & $\mathrm{O}_{\mathrm{f}}$ in Eq. 3 & - & 0.1 & 0 & 0.1 \\
\hline \multirow[t]{4}{*}{ Wastewater } & $\begin{array}{l}\text { MCF for domestic sewage treated by } \\
\text { WTPs }\end{array}$ & - & 0.165 & 0.1 & 0.3 \\
\hline & $\begin{array}{l}\text { MCF for industrial wastewater treated } \\
\text { by WTPs }\end{array}$ & - & 0.467 & 0.2 & 0.5 \\
\hline & $\begin{array}{l}\text { MCF for wastewater discharged into } \\
\text { rivers, lakes or ocean }\end{array}$ & - & 0.1 & 0 & 0.2 \\
\hline & $\mathrm{B}_{0}$ in Eq. 4 & $\mathrm{kgCH}_{4} / \mathrm{kgCOD}$ & 0.25 & 0.175 & 0.325 \\
\hline
\end{tabular}


Table S3. Total $\mathrm{CH}_{4}$ emissions of each province in four snapshot years $(1980,1990,2000$ and 2010). Values are given in $\mathrm{Tg} \mathrm{CH}_{4} \mathrm{yr}^{-1}$ (mean [min-max]).

\begin{tabular}{|c|c|c|c|c|}
\hline & \multicolumn{4}{|c|}{$\mathrm{CH}_{4}$ emissions $\left(\mathrm{Tg} \mathrm{CH}_{4} \mathrm{yr}^{-1}\right)$} \\
\hline & 1980 & 1990 & 2000 & 2010 \\
\hline Beijing & $0.1[0.1-0.1]$ & $0.1[0.1-0.2]$ & $0.2[0.1-0.2]$ & $0.2[0.1-0.2]$ \\
\hline Tianjin & $0.1[0.0-0.1]$ & $0.1[0.1-0.1]$ & $0.1[0.1-0.2]$ & $0.2[0.2-0.5]$ \\
\hline Hebei & $0.5[0.4-0.7]$ & $0.8[0.5-1.0]$ & $1.3[1.0-1.7]$ & $1.5[1.1-2.0]$ \\
\hline Shanxi & $0.7[0.5-0.8]$ & $1.5[1.1-1.8]$ & $1.3[1.0-1.7]$ & $3.3[2.6-4.2]$ \\
\hline Inner Mongolia & $0.5[0.4-0.6]$ & $0.7[0.5-0.9]$ & $0.9[0.7-1.2]$ & $3.3[3.0-3.8]$ \\
\hline Liaoning & $0.7[0.6-1.0]$ & $1.0[0.8-1.3]$ & $1.1[0.8-1.4]$ & 1.4 [1.1-1.7] \\
\hline Jilin & $0.4[0.3-0.4]$ & $0.6[0.4-0.7]$ & $0.8[0.6-1.0]$ & $1.1[0.9-1.4]$ \\
\hline Heilongjiang & $0.8[0.6-1.2]$ & $1.4[1.1-2.0]$ & $1.5[1.1-2.1]$ & $2.0[1.6-2.7]$ \\
\hline Shanghai & $0.2[0.1-0.2]$ & $0.1[0.1-0.2]$ & $0.1[0.1-0.2]$ & $0.2[0.1-0.2]$ \\
\hline Jiangsu & $1.0[0.8-1.2]$ & $1.0[0.7-1.2]$ & $1.0[0.7-1.3]$ & $1.1[0.7-1.4]$ \\
\hline Zhejiang & $1.3[0.8-1.6]$ & $1.2[0.8-1.5]$ & $0.8[0.6-1.0]$ & 0.7 [0.5-0.9] \\
\hline Anhui & $0.9[0.8-1.1]$ & $1.2[1.0-1.4]$ & 1.4 [1.1-1.7] & 1.5 [1.2-1.8] \\
\hline Fujian & $0.8[0.6-1.1]$ & $0.8[0.6-1.0]$ & $0.6[0.5-0.8]$ & $0.6[0.5-0.8]$ \\
\hline Jiangxi & $1.5[1.1-2.0]$ & $1.6[1.1-2.1]$ & $1.4[1.0-1.8]$ & $1.6[1.1-2.0]$ \\
\hline Shandong & $0.8[0.5-1.2]$ & $1.3[0.9-1.8]$ & $2.0[1.4-2.6]$ & 2.1 [1.5-2.8] \\
\hline Henan & $0.9[0.7-1.1]$ & $1.6[1.3-2.0]$ & $2.2[1.7-2.7]$ & 2.7 [2.3-3.2] \\
\hline Hubei & $1.0[0.9-1.2]$ & $1.2[1.0-1.4]$ & $1.1[0.8-1.3]$ & $1.1[0.8-1.3]$ \\
\hline Hunan & $2.1[1.5-2.8]$ & 2.2 [1.6-2.9] & $2.1[1.5-2.7]$ & 2.4 [1.9-2.9] \\
\hline Guangdong & 2.1 [1.4-2.7] & 1.8 [1.2-2.3] & $1.6[1.1-2.2]$ & $1.6[1.0-2.2]$ \\
\hline Guangxi & 1.5 [1.0-1.9] & $1.6[1.1-2.1]$ & $1.6[1.1-2.1]$ & 1.4 [0.9-1.8] \\
\hline Hainan & $0.0[0.0-0.0]$ & $0.3[0.2-0.3]$ & $0.3[0.2-0.3]$ & $0.2[0.2-0.3]$ \\
\hline Chongqing & $0.0[0.0-0.0]$ & $0.0[0.0-0.0]$ & $0.7[0.6-0.8]$ & 1.1 [1.0-1.2] \\
\hline Sichuan & $2.8[2.5-3.1]$ & 3.3 [2.9-3.7] & $2.5[2.1-2.8]$ & $3.0[2.6-3.4]$ \\
\hline Guizhou & $0.9[0.8-1.0]$ & 1.3 [1.1-1.5] & 1.3 [1.2-1.5] & 2.9 [2.7-3.3] \\
\hline Yunnan & $1.1[0.9-1.2]$ & 1.3 [1.1-1.5] & $1.4[1.2-1.6]$ & 2.0 [1.7-2.2] \\
\hline Tibet & $0.3[0.3-0.5]$ & $0.4[0.3-0.5]$ & $0.4[0.3-0.5]$ & $0.4[0.3-0.6]$ \\
\hline Shaanxi & $0.3[0.3-0.4]$ & $0.5[0.4-0.6]$ & $0.5[0.4-0.7]$ & $2.3[2.2-3.1]$ \\
\hline Gansu & $0.2[0.2-0.3]$ & $0.4[0.3-0.5]$ & $0.4[0.3-0.6]$ & 0.7 [0.6-0.9] \\
\hline Qinghai & $0.4[0.3-0.5]$ & $0.4[0.3-0.6]$ & $0.4[0.3-0.5]$ & 0.5 [0.4-0.8] \\
\hline Ningxia & $0.1[0.1-0.1]$ & $0.1[0.1-0.2]$ & $0.2[0.2-0.2]$ & $0.5[0.4-0.5]$ \\
\hline Xinjiang & $0.4[0.3-0.5]$ & $0.5[0.4-0.7]$ & $0.8[0.6-1.1]$ & $1.4[1.2-2.3]$ \\
\hline
\end{tabular}


Figure S1. The annual output of biogas ( $\mathrm{Tg} \mathrm{CH}_{4} / \mathrm{yr}$ ) from 1980 to 2010. The biogas data is assumed a linear increase from 1980 to 1996. The biogas data from 1996 to 2010 is available from Feng et al. (2012).

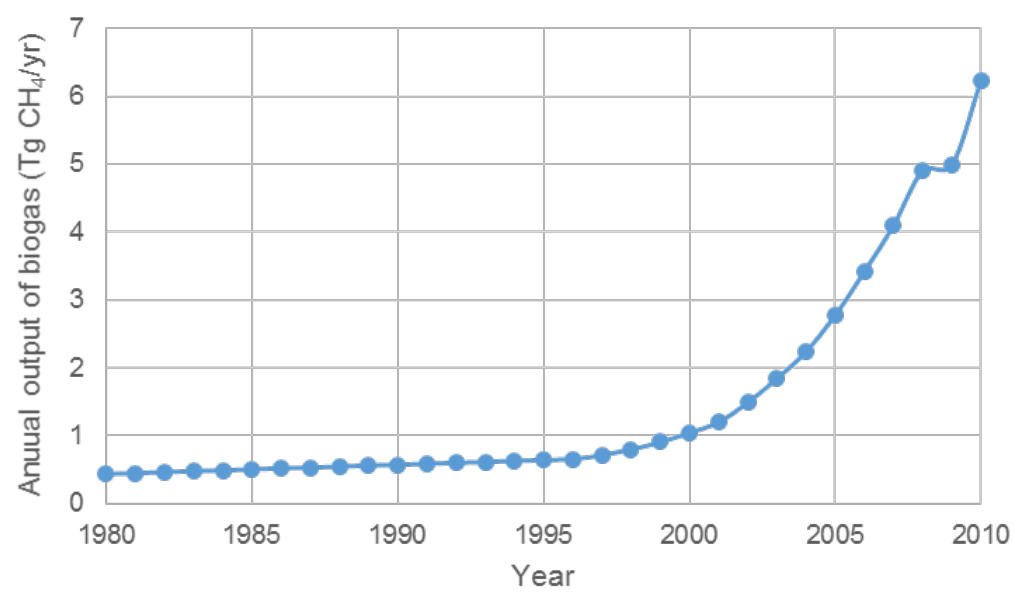


Figure S2. The total chemical fertilizer input and fraction of rice paddy with organic matter (OM) input from 1980 to 2010 in China.

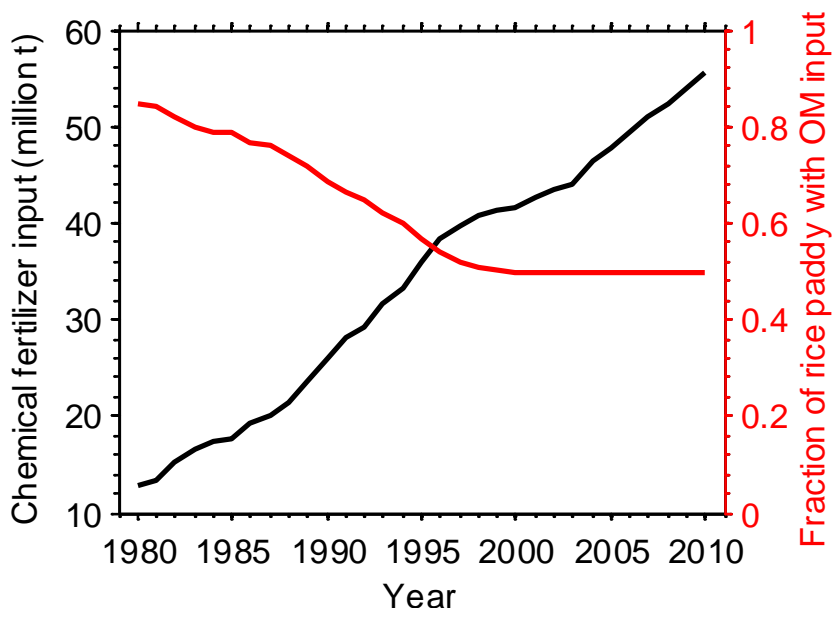


Figure S3. The relationship between Gross Domestic Product (GDP) and the fraction of municipal solid waste (MSW) treated by landfills.

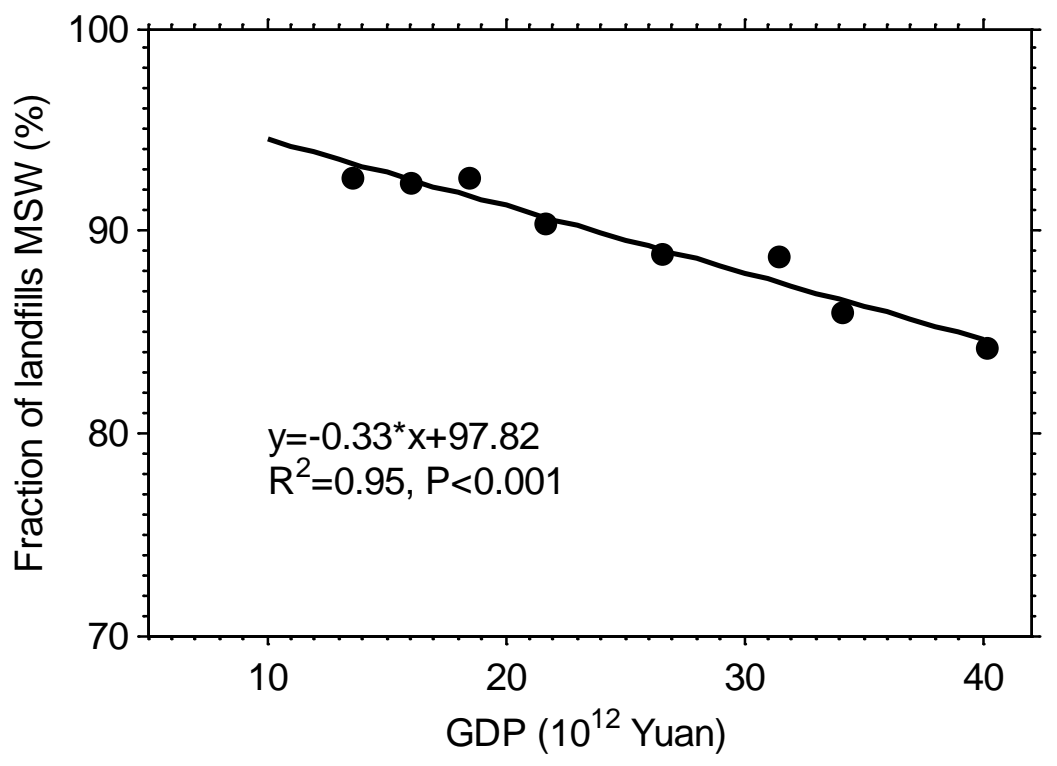


Figure S4. (a) The relationship between Gross Domestic Product (GDP) and COD in domestic sewage treated by wastewater treatment plants (WTPs). (b) The relationship between population and the total of COD in domestic sewage.
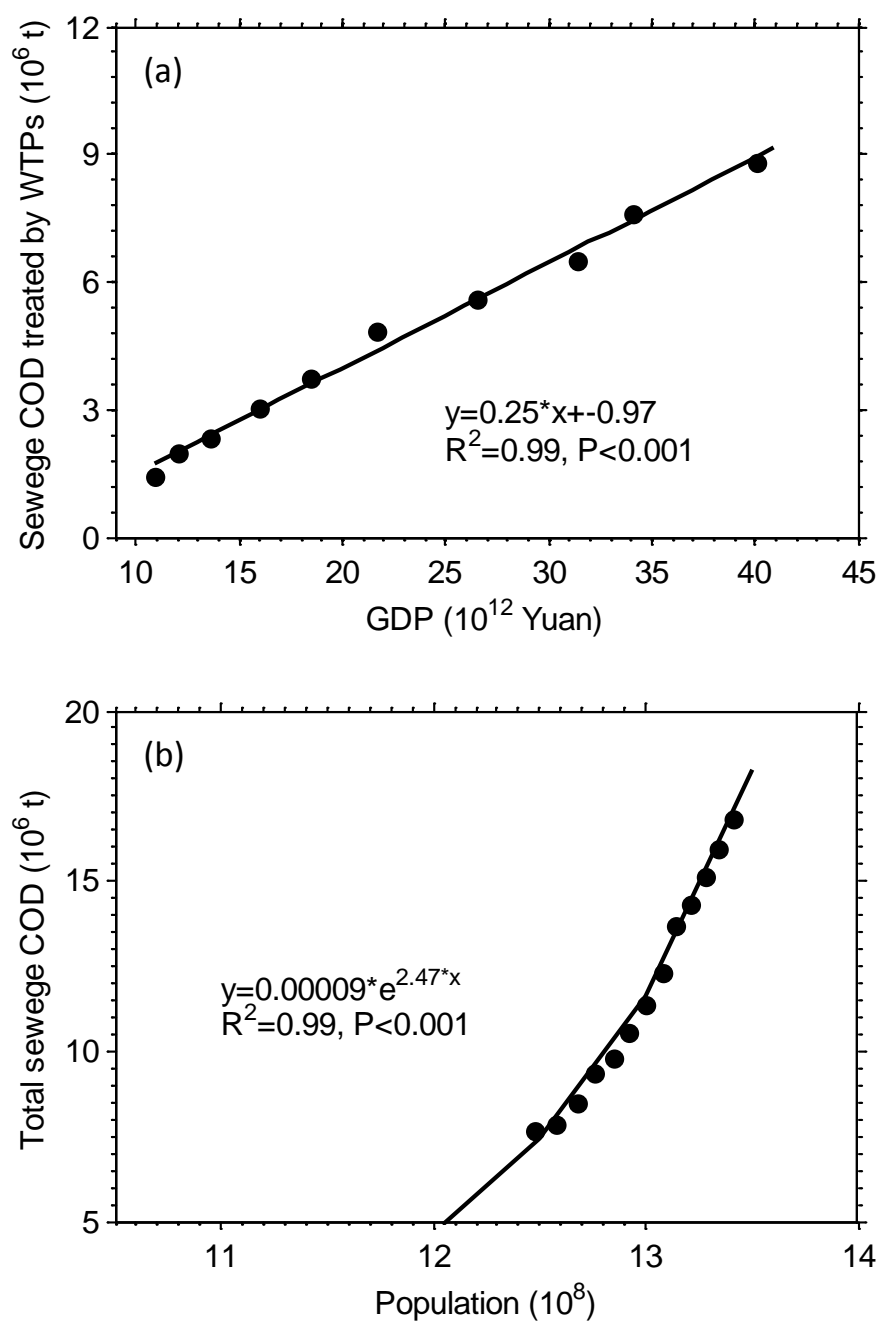
Figure S5. (a) The relationship between industrial Gross Domestic Product (GDP) and COD in industrial wastewater treated by wastewater treatment plants (WTPs). (b) The relationship between GDP and the fraction of COD in industrial wastewater discharged into rivers, lakes and ocean to the total COD in industrial wastewater.
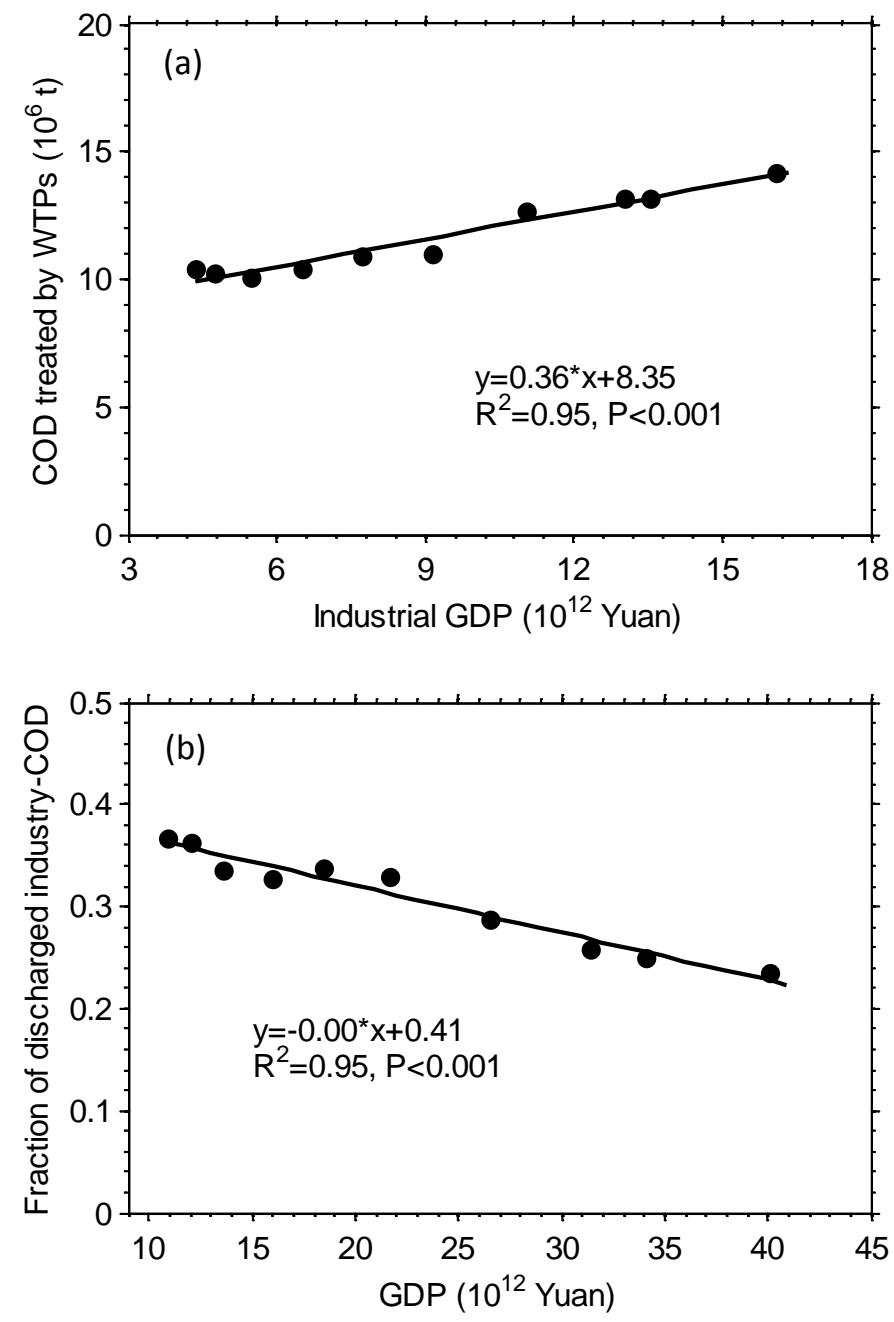
Figure S6. (a) Provinces and (b) regions in Mainland China.
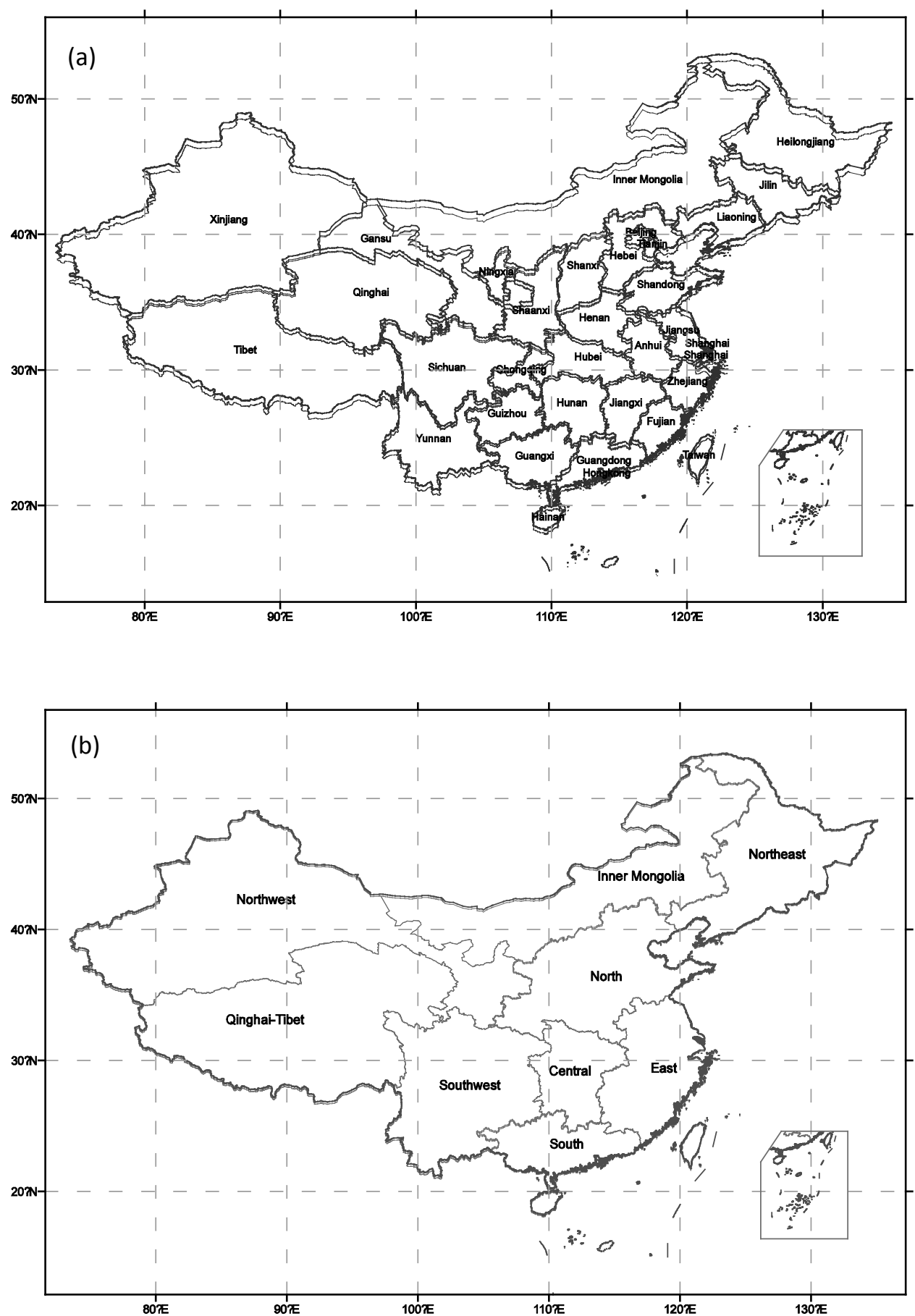